\title{
Review: 'Pseudo'- syndromes in cardiology
}

\author{
Mishra $\mathrm{A}^{1}$, Mishra $\mathrm{S}^{2}$ and Mishra JP2* \\ ${ }^{1}$ Georgetown University, Washington, DC, USA \\ ${ }^{2}$ Upstate Cardiology, Summit St, Batavia, New York, USA
}

\begin{abstract}
The term 'pseudo' means 'false', 'pretended', 'unreal', or 'sham' and it is likely to be of Greek origin, pseudes means false. There are a number of 'pseudo' terms and syndromes that we see in the practice of cardiology. Even though the meaning of pseudo is unreal or sham, however these syndromes are true entities as described below. These terms appear more 'mimicking' a diagnosis than being truly 'sham'!

We attempted to put together most of the 'pseudo'-diagnoses under one heading for reference and convenience. However, every condition cannot be described here in full details and the references are available for further studies.
\end{abstract}

\section{Takotsubo cardiomyopathy (Pseudo acute myocardial infarction)}

This condition was first described in patients in Japan in 1990 presenting and mimicking as acute coronary syndrome (ACS) with chest pains, ST changes on ECG and mild cardiac enzyme elevation consistent otherwise with acute myocardial infarction (AMI). Imaging studies show LV apical ballooning and therefore being called Takotsubo cardiomyopathy (meaning "Octopus pot") However, coronary angiography in these patients will at best reveal mild atherosclerosis [1].

This condition is most often seen in elderly females and mostly right after a significant emotional stress. Prognosis is generally favorable with significant improvement after optimal medical therapy.

\section{Modified Mayo Clinic Criteria for its diagnosis [2]}

- Transient hypokinesis/akinesis of LV apex or midsegment, the regional wall motion abnormalities extending beyond a single epicardial distribution and a stressful trigger mostly present.

- Absence of any obstructive coronary artery disease.

- New ECG changes with either ST-segment elevation and/or T-wave inversion.

- Absence of pheochromocytoma or myocarditis.

This condition therefore presents itself mimicking as (pseudo) myocardial infarction.

\section{Pseudo-wellens' syndrome}

Wellens' syndrome is a pattern of ECG changes mostly T wave inversion in the setting of angina and is associated with critical, proximal left anterior descending artery (LAD) stenosis. This is a pre-infarction stage and with continued medical therapy and without intervention/ stent placement is very likely to lead to myocardial infarction or death within a few days [3]

\section{Criteria for Wellens' syndrome include}

- History of anginal chest pains.
- Minimal or no cardiac enzyme elevation.

- No significant ST-elevation $(<1 \mathrm{~mm})$.

- No pathological Q waves in precordial leads.

- Deeply inverted or biphasic T waves in V2-3 or sometimes V4-6. These changes seen during pain-free period.

Similar changes can be seen in the setting of chest pains along with above noted criteria, however without having any critical LAD obstruction (Pseudo-Wellens' syndrome). The presumed theory of these chest pains and ST changes are likely due to coronary vasospasm. These kinds of cases have been reported after illicit drug use [4] e.g. marijuana [5] and also with myocardial bridging spasm [6].

\section{Pseudo-pacemaker syndrome due to first-degree atrioventricular block}

First-degree atrioventricular (AV) block is defined as a delay in transmission of an impulse from the atria to the ventricles resulting in a PR interval longer than $200 \mathrm{msec}$. This can be due to anatomical or functional impairment in the conduction system. First-degree AV block can be seen in healthy children, athletes and otherwise healthy adults and for most part, it is asymptomatic.

However, sometimes when first-degree AV block is markedly prolonged, it can result in atrial contraction occurring very close to the ventricular systole causing hemodynamic changes thereby decrease in cardiac output and heart failure thus mimicking a pacemaker syndrome so called 'pseudo-pacemaker syndrome' [7]. Cases have been reported with systemic hypotension, increased pulmonary arterial pressures and cannon A waves in jugular veins. If a patient has dual AV nodal physiology with preferential antegrade slow pathway conduction, one can present with similar symptoms. Keeping the same principle in

${ }^{\star}$ Correspondence to: JP Mishra, Upstate Cardiology, Summit St, Batavia, New York, USA, E-mail: jpmish@gmail.com

Key words: pseudes, myocardial, Takotsubo, cardiomyopathy, infarction

Received: July 13, 2018; Accepted: July 24, 2018; Published: July 26, 2018 
mind, if the fast pathway is unintentionally injured during catheter ablation for $\mathrm{AV}$ nodal reentrant tachycardia, the symptoms of pseudopacemaker syndrome can be elicited [8].

The treatment of this symptomatic condition is dual chamber pacemaker implantation (to restore a physiologic AV interval) and it is recommended as level IIA, evidence B as per the device guidelines [9].

Also, the slow pathway modification of markedly prolonged symptomatic first-degree AV block in the setting of dual AV nodal physiology is being recommended as a new alternative mode of management as reported recently [8].

\section{Pseudo-myocardial infarction}

There are a number of conditions where ECG changes appear abnormal and patients mimic having myocardial infarction. Some of these ECG changes are reported below [10]:

1. In left ventricular hypertrophy, there is often a QS pattern or poor $\mathrm{R}$ wave progression in the right precordial leads suggesting anterior myocardial infarction. The secondary ST-changes may be mistaken as a current of injury.

2. In pulmonary emphysema, the $\mathrm{R}$ wave in precordial leads and sometimes mid-precordial leads become quite small or absent giving an appearance of anterior myocardial infarction. These changes are due to the vertical heart secondary to a low-lying diaphragm and the intervention of hyperinflated lungs.

3. Similar ECG pattern can also be seen in pneumothorax with reduced QRS voltage and QS pattern in right precordial leads.

4. In pulmonary embolism, the Q waves in lead III (as part of the S1Q3 pattern), and sometimes in lead aVF that are accompanied by ST-segment and T-wave changes are often interpreted as inferior myocardial infarction.

5. In hypertrophic cardiomyopathy, abnormal Qs are often seen especially in the left precordial leads most likely due to ventricular septal hypertrophy.

6. Pseudoinfarction pattern can be seen due to myocardial fibrosis in patients with dilated cardiomyopathy, progressive muscular dystrophy, amyloidosis and the tumors of the heart.

7. QS pattern are often seen in the right precordial leads in patients with left bundle branch block (LBBB) in the absence of myocardial infarction.

8. Left anterior hemiblock is occasionally associated with small Qs in the precordial leads that mimic anterior myocardial infarction.

9. The delta waves in wolff-Parkinson-White syndrome (WPW) are frequently interpreted as abnormal Qs of myocardial infarction (The issue is further discussed later in this chapter).

10. Pheochromocytoma may be associated with ECG changes mimicking as ischemic heart disease.

11.Intracranial bleeding, hyperkalemia, pancreatitis and acute pericarditis may present with ECG changes suggestive of myocardial infarction.

\section{Wolff-parkinson-white syndrome (wpw): pseudo myocardial infarction}

There are some ECG changes taking place in WPW syndrome that simulate myocardial infarction. The morphology of these pseudo
ECG patterns depends on the anatomical location of the bundle of Kent accessory pathway on atrioventricular (AV) ring. The rightsided accessory pathway may cause prominent R-waves in the right precordial leads simulating posterior myocardial infarction. Likewise, the left accessory pathway may cause Qs in lateral limb leads (I and aVL) simulating high lateral myocardial infarction.

The posterolateral accessory pathways, both the right- and the left-sided may cause Qs inferiorly mimicking inferior wall myocardial infarction [11].

\section{Pseudosyncope, psychogenic}

Transient loss of consciousness is quite common and one of the most common presentation to the emergency room. Major reasons for passing out are: syncope, epileptic seizures and psychogenic syncope.

There are inherent socioeconomic and medicolegal problems related to making a diagnosis of syncope versus psychogenic pseudosyncope [12].

\section{Pseudo r' waves in lead vl}

ECG recognition of the P' wave during tachycardia is very useful in the diagnosis of supraventricular tachycardias. In atrioventricular nodal reentrant tachycardia (AVNRT), no discrete P' waves are observed on ECG and pseudo r' deflection in lead V1 (pseudo $\mathrm{r}^{\prime}$ ) is commonly seen (90\%). It is an upward deflection in the terminal portion of the QRS complex during tachycardia and not seen during sinus rhythm. Pseudo $r^{\prime}$ deflection in lead V1 is a highly sensitive indicator of AVNRT and appears to result from the activation of the superolateral aspect of the right atrium [13].

\section{Pseudo left axis deviation (the s1s2s3 syndrome)}

Many patients with pulmonary emphysema will have right axis deviation on the ECG, however there are some patients with this disease entity who have left axis deviation in the absence of clinical coronary artery disease, systemic hypertension or other causes of myocardial failure. This kind of left axis deviation is called pseudo left axis deviation (S1S2S3 syndrome).

The most frequent ECG changes seen in chronic airway obstruction: 1. Inferior P axis. 2. P-pulmonale. 3. Right axis deviation. 4. Low voltage.

The incidence of left axis deviation in chronic airway obstruction has been reported to be $2-13 \%$. The exact cause of this ECG finding is unknown but one presumed mechanism is related to a marked reduction in the electrical conductance in the emphysematous lungs and to the thoracic deformity causing the heart to shift leftward and posterior in position. These ECG changes are likely to be seen in severe chronic airway obstruction cases [14].

\section{Pseudo-atrial flutter (“tup” syndrome)}

Some cases of palpitations and tachycardia are seen in the cardiology office or the emergency room and the diagnosis of atrial flutter is made. Many a times these ECGs diagnoses turn out to be something else otherwise called pseudo-atrial flutter as shown by two following cases. Many a times hypokalemia, hypomagnesemia or Parkinsonian tremors are believed to be the cause of the problem. In the 2nd case, the 'flutter' waves on 12 lead ECG are seen mostly in the limb leads. After documenting in the EP lab that there is only sinus rhythm, the leads were placed again from the torso on to the limb leads and those 'flutter' waves were reproduced $[15,16]$ ! 
'Flutter' waves reproduced after the completion of the EP study when the leads were placed on the limb leads!

\section{Pseudo brugada pattern}

Brugada syndrome is characterized by a hereditary anomaly in the sodium ion channel (mutation of the SCN5A gene) identified by a wide QRS associated with ST segment/J point elevation and T wave inversion in the right precordial leads. Ventricular tachycardia and sudden cardiac death can be associated with this condition.

Sodium-channel blocking drugs, lithium, cocaine, hypothermia and electrolyte imbalances eg hyperkalemia can trigger Brugada pattern.

A few cases of pseudo Brugada syndrome have been reported following hyperkalemia with wide complex tachycardia and absence of $\mathrm{P}$ waves without any other ECG changes seen with hereditary Brugada syndrome. The electrophysiologic mechanisms underlying ECGs of patients with the hyperkalemic Brugada pattern are unknown. It is possible that severe extracellular hyperkalemia which results in inactivation of the cardiac sodium channel may also cause a transient Brugada pattern in susceptible patients.

There is a genetic variant of the Brugada syndrome called pseudopseudo-Brugada syndrome.

\section{Pseudo 2:1 av block}

Pseudo 2:1 AV block has been initially described in a patient with typical junctional premature systoles, blocked premature $\mathrm{P}$ waves of retrograde fashion, a sudden prolongation of the PR interval and occasional block of normally occurring of sinus $\mathrm{P}$ waves.

In congenital long QT syndrome (LQTS), sometimes because of extreme prolongation of ventricular action potential duration, two forms of alternating activity of the heart may occur: a pseudo 2:1 AV block and a $\mathrm{T}$ wave alteration both of which are rate dependent. The pseudo 2:1 AV block is due to extreme prolongation of ventricular refractoriness. The $\mathrm{T}$ wave alteration reflects the fact that the rate dependence of action potential duration differs in degree or magnitude in different layers of ventricular wall.

\section{Pseudonormal diastolic filling}

Assessment of LV diastolic function is considered as important as the assessment of LV systolic function in cardiology. It is quite comprehensive and various modalities are available for this evaluation.

$2 \mathrm{D}$-echocardiography is one non-invasive and easily available technique for LV diastolic function assessment. The most important modality to assess diastolic function is the mitral inflow signal. The mitral inflow signal measures the pressure difference between the atrium and the ventricle and thus helps define diastolic dysfunction.

Diastolic dysfunction: The detailed discussion can be found in different references. Just to summarize 4 phases of diastolic dysfunction here:

Grade I: Impaired relaxation

Grade II: Pseudonormal filling pattern

Grade III: Reversible restrictive filling pattern

Grade IV: Irreversible restrictive filling pattern.

\section{Pseudo-anginal pains}

There are many causes of chest pains that will mimic anginal pains including esophageal, arthritic, pleural, pericardial, and so on.
The esophageal causes of pseudo-anginal pains need to be mentioned here as those are quite common, could be very debilitating and will likely need GI evaluation, assurance and treatment. Some of these are:

Diffuse esophageal spasm

Nutcracker esophagus

Segmental non-peristalsis in the esophagus

Segmental high amplitude contractions

Hypertensive lower esophageal sphincter

Achalasia

Acid-induced chest pains (GERD).

\section{Pseudo-right heart overload}

Congenital absence of pericardium (CAP) is a very rare cause of atypical anginal symptoms or dyspnea. Its prevalence is vastly underestimated, mostly due to lack of symptoms and poorly known diagnostic criteria.

There are at least 6 types of CAP have been reported in the literature based on the embryology and anatomy.

These are:

Total aplasia of the pericardium (9\%). (complete and partial)

Diaphragmatic pericardial defects (17\%).

Agenesis of the right pleuropericardial membrane (4\%).

Agenesis of the left pleuropericardial membrane (70\%). (complete and partial).

Partial defects of the left pleuropericardial membrane are the most recognized defects, usually located in the upper part of the left pericardium and enable herniation of the left atrial appendage (LAA) seen on a chest $\mathrm{x}$-ray.

Palpitations, syncope or sudden cardiac death are the features noted in the partial/localized forms, however the total absence of pericardium can have no symptoms at all.

Some kinds of angina will be noted in most of these patients. The mechanism of chest pains remains speculative and elusive.

The ECG changes reported are: right axis deviation, incomplete right bundle branch leftward displacement of the transition zone in the precordial leads.

Chest x-ray will classically show levoposition of the heart without tracheal deviation. The left border is elongated and flattened with prominence of the main pulmonary artery which is separated from the aortic knob by a radiolucent zone giving a characteristic silhouette (Snoopy sign).

Interposition of lung tissue between the heart and the left diaphragm may be better seen on tomogram or perfusion lung scan.

\section{The echocardiographic features}

the right ventricular overload, right ventricular enlargement and abnormal septal motion. In 2D-echocardiography, the most striking finding is failure to obtain the standard views by the usual acoustic windows. 


\section{Cardiac MRI}

Presence of normal pericardium as a thin line of uniform lowsignal intensity separating the high-signal intensity fat within the epicardial and pericardial fat. In case of an absent left pericardium, MRI will classically show: 1 . Abnormal heart position, completely moved into the left hemithorax 2. Abnormal and pathognomonic presence of lung parenchyma in the usually lung-free aortopulmonary space 3. No visualization of the left pericardium together with an indistinct posterior cardiopulmonary interface.

\section{Treatment}

Large or total left-sided defects are usually benign and need no treatment. In some cases of intense pain, surgical reconstruction of the pericardium either by allograft or by means of Gore-Tex material has been proposed and generally reduces the intensity of pains.

\section{Pseudo-tumor of chf}

\section{Pulmonary Pseudo-tumors}

Most often this term is used to denote focal collections of fluid trapped in the pleural fissures. There are other terms used for condition: round atelectasis, pulmonary inflammatory pseudotumor, epicardial fat pad, mucoid impaction and calcifying fibrous pseudotumor (CFPT) of lung.

The fluid collected is usually small and asymptomatic. Generally, no direct treatment is warranted.

\section{"Pseudo-heart disease" in straight back syndrome}

Straight Back Syndrome (SBS) is a rare congenital chest wall deformity where the back curvature of the thoracic spine in absent; instead it is straight. It narrows the space between the posterior aspect of the manubrium and the anterior thoracic spine. It might be associated with pectus excavatum, sometimes with accompanied tracheal compression and dyspnea.

Many a times this condition is associated with mitral valve prolapse and 'pseudo-heart disease': atypical chest pains, palpitation, dyspnea and pulmonary hypertension. The surgical intervention will depend upon the extent and severity of symptoms.

\section{Pseudonormal T waves}

In patients with inverted $\mathrm{T}$ waves at baseline, the evolution of an upright $\mathrm{T}$ waves during exercise ('pseudonormalization') has been considered to represent ischemia by some experts, however others believe it to be non-significant.

In addition, the development of pseudonormalization of $\mathrm{T}$ waves after recent myocardial infarction has been controversial as well during treadmill exercise, during spontaneous angina or while Holter monitoring. It has been shown before that exercise-induced ST-segment elevation in infarct-related leads is almost invariably associated with the presence of metabolically active myocardium, however the relationship between these ST and T changes during exercise and LV function recovery is unclear.

In one study, low dose dobutamine stress echocardiography was performed in patients with Q-wave myocardial infarction and it was reported that the contractile reserve was higher in the area with $\mathrm{T}$ wave pseudonormalization or ST-segment elevation that those area without any changes on the ECG. Similar findings were reported by Mobilia et al stating that these ECG changes were the markers of viable myocardium and that was corroborated by PET (positron emission tomography). However, another study by Bodi et al did not find any strong relationship between the ECG changes (ST-segment elevation and $\mathrm{T}$ wave normalization with treadmill) and functional recovery.

\section{Pseudo-aneurysms}

Pseudo-aneurysms of the vessels.

Pseudo-aneurysm of the LV Wall.

Pseudo-aneurysms of the vessels

False aneurysms (pseudoaneurysm): when there is a discontinuity in the vessel wall thereby the blood leaks through the wall and is contained by the adventitia or surrounding perivascular soft tissue. A direct communication of blood flow exists between the vessel lumen and the aneurysm lumen through the hole in the vessel wall. The risk of rupture is higher than that of a true aneurysm of comparable size due to poor support of the pseudo-aneurysms and therefore mostly needing the treatment.

These can involve any arterial segment eg:

- aortic pseudoaneurysm: traumatic aortic pseudoaneurysm

- femoral artery pseudoaneurysm: relatively common site due to femoral punctures

- carotid artery pseudoaneurysm

- visceral arterial pseudoaneurysm

- hepatic arterial pseudoaneurysm

- gastroduodenal arterial pseudoaneurysm

- splenic arterial pseudoaneurysm.

- pre-pancreatic pseudoaneurysm

- renal arterial pseudoaneurysm

- peripheral arterial (limb) pseudoaneurysm

- brachiocephalic artery pseudoaneurysm.

\section{Pseudo-aneurysm of the lv wall}

Left ventricular pseudoaneurysms are false aneurysms that result from contained myocardial rupture and are a rare complication of a myocardial infarction (MI). These should not be confused with left ventricular aneurysms which are true aneurysms containing all the layers (endocardium, myocardium, and epicardium).

Left ventricular pseudoaneurysm formation typically results from rupture of the myocardial free wall (most frequent 3-7 days post-MI). The cardiac rupture is contained by adherent pericardium or scar tissue.

The pseudoaneurysm wall consists only of the epicardium, pericardium and haematoma. In contrast, a left ventricular aneurysm has a wall of scarred myocardium and endocardium.

It most commonly involves inferior/posterior wall and sometimes lateral wall of the left ventricle. 2D-echocardiography and MRI will be helpful to make the diagnosis and most patients will need surgical intervention.

\section{Pseudo aortic stenosis (low grade as)}

Low gradient aortic stenosis is defined as an aortic valve area $<1 \mathrm{~cm}^{2}$ and mean gradient $<40 \mathrm{mmHg}$, and there could be different patterns 
of low-gradient aortic stenosis. The classic low-flow, low-gradient AS, which is the form of low-gradient AS that is associated with reduced ejection fraction, is called D2 stage in the guidelines. The paradoxical low-flow, low-gradient AS (D3 stage) is characterized by low-flow state $\left(\mathrm{SVi}<35 \mathrm{~mL} / \mathrm{m}^{2}\right)$ despite preserved LVEF $(>50 \%)$. The low flow is due to marked LV concentric remodeling with a small LV cavity size, diastolic dysfunction, and reduced longitudinal shortening. The third type of low gradient AS is characterized by normal flow and is not addressed in the guidelines (Figure 1-5).

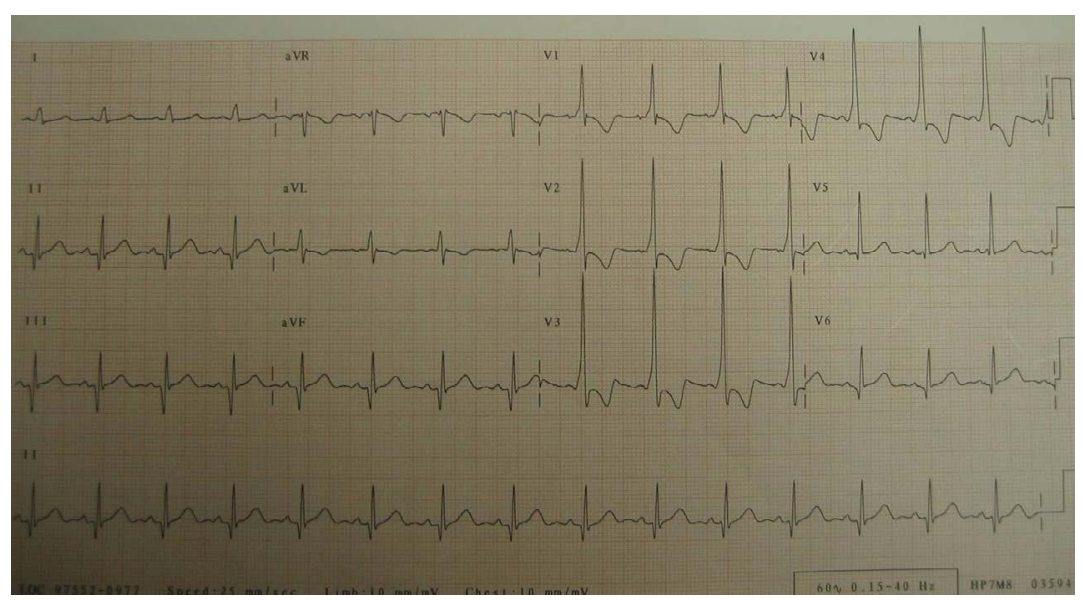

Figure 1. WPW syndrome mimicking infero-lateral MI

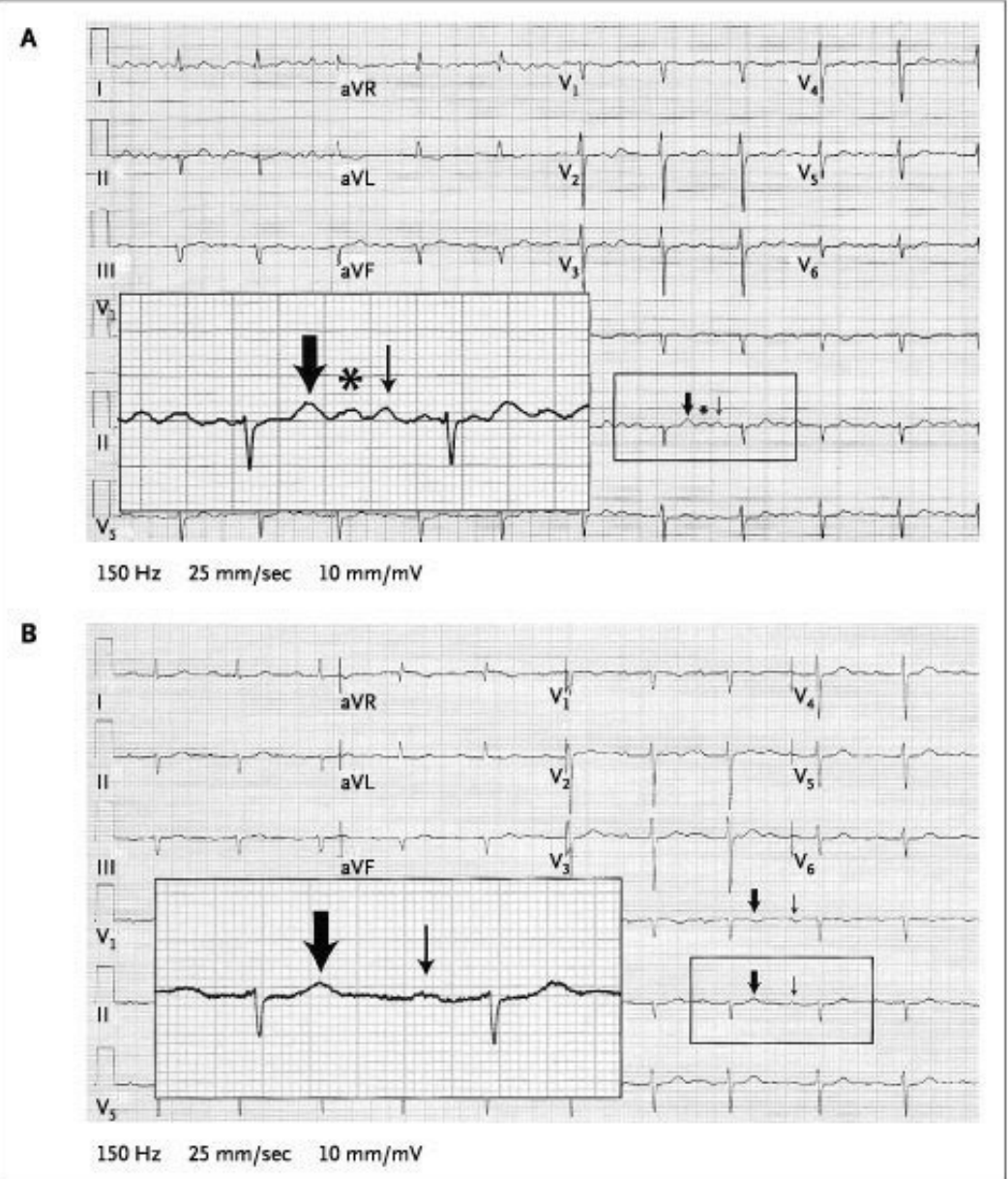

Figure 2. A 77-year-old man with hypertension on hydrochlorothiazide was found to have hypokalemia at $2.6 \mathrm{mE} / \mathrm{L}$. (A) 4:1 atrial flutter was diagnosed (Thick arrow: T, asterisk: U, and thin arrow: P) (B) during EP study, only Ps were seen 


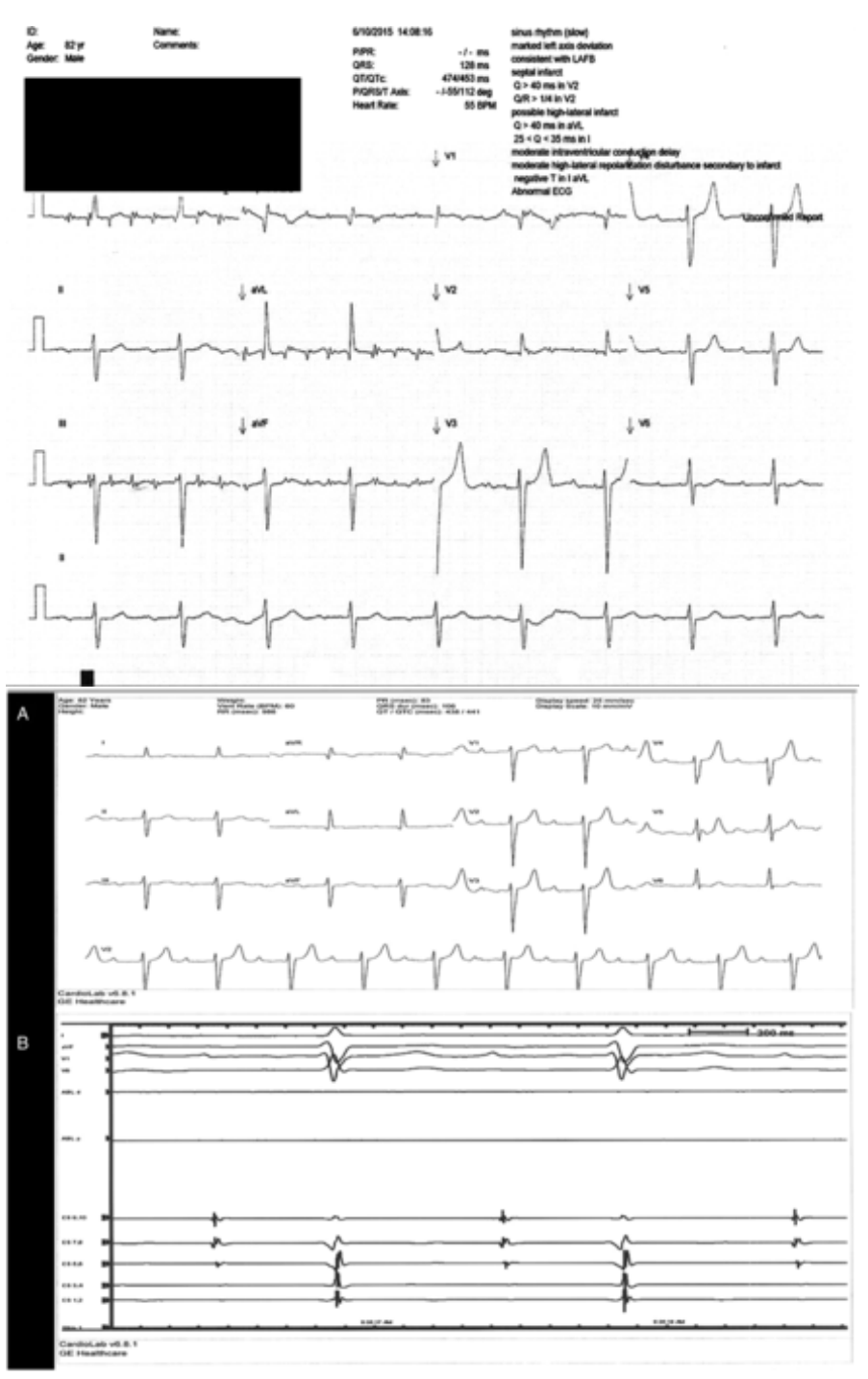

Figure 3. An 82-year-old man with bioprosthetic valve for severe aortic stenosis was found to have tachycardia with 3:1 response on his ECG. In the EP lab, only sinus rhythm. Pseudo atrial flutter was diagnosed

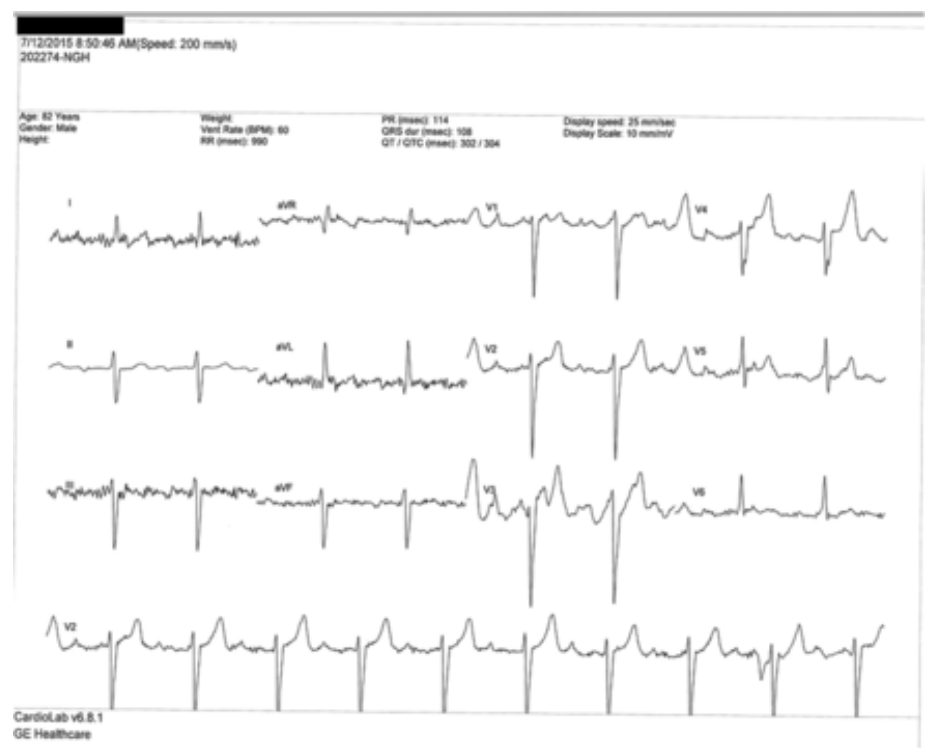

Figure 4. 'Flutter' waves reproduced after the completion of the EP study when the leads were placed on the limb leads! 

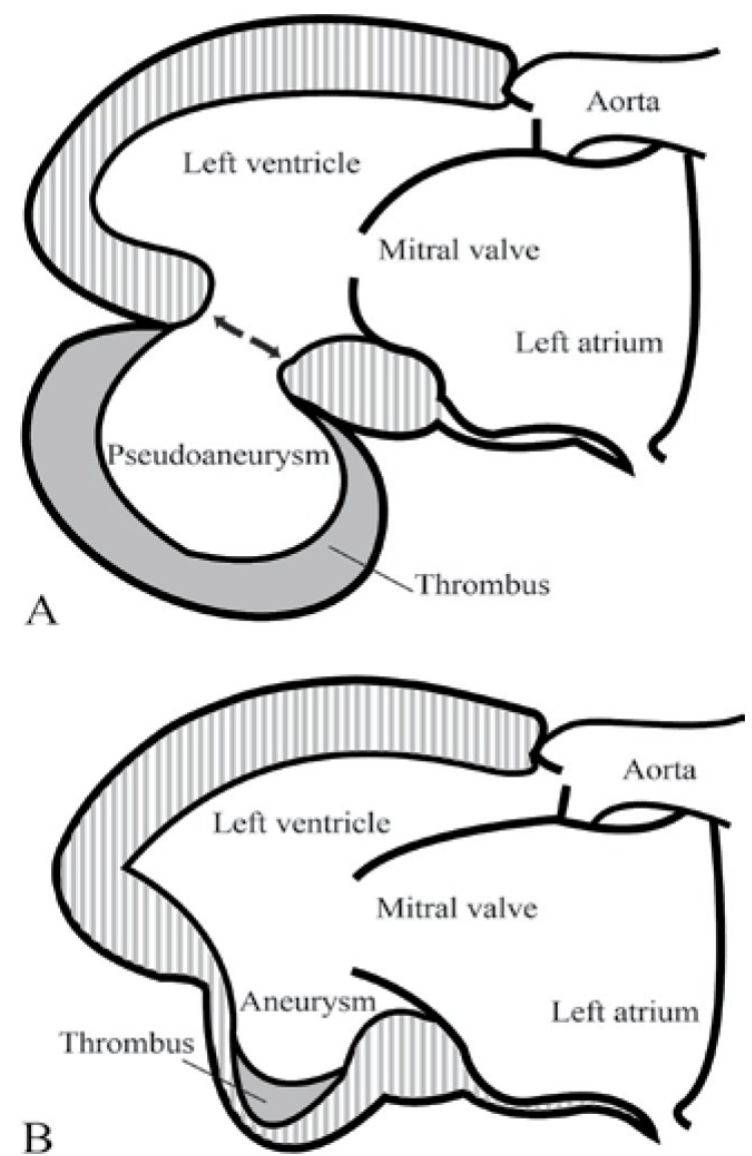

Figure 5. Inferior-posterior LV wall showing pseudoaneurysm (A) vs aneurysm (B)

\section{Conclusion}

Most of the 'pseudo' terms/conditions are discussed under one topic that are commonly seen and practiced in cardiology. However, the goal was not to present the detailed discussion of every disease mentioned here. We would sincerely request to go to the references for more information on any individual disease state.

\section{References}

1. Terefe YG, Niraj A, Pradhan J, Kondur A, Afonso L (2007) Myocardial infarction with angiographically normal coronary arteries in the contemporary era. Coron Artery Dis 18: 621-626. [Crossref]
2. Kawai S, Kitabatake A, Tomoike H, Takotsubo Cardiomyopathy Group (2007) Guidelines for diagnosis of takotsubo (ampulla) cardiomyopathy. Circ J 71: 990-992. [Crossref]

3. De Zwaan C, Bar Fw, Wellens HJ (1982) Characteristic electrocardiographic pattern indicating a critical stenosis high in left anterior descending coronary artery in patients admitted because of impending myocardial infarction. Am Heart $J$ 103:730-736. [Crossref]

4. Dhawan SS (2008) Pseudo-Wellens' syndrome after crack cocaine use. Can $J$ Cardiol 24: 404. [Crossref]

5. Ferreras ML, Das A, Okwuosa T (2017) Pseudo-Wellens' syndrome after heavy Marijuana use. Cleve Clin J Med 84: 590-591. [Crossref]

6. Kaplanis I, Michas G, Arapi S, Thomopoulos T, Stougiannos P, et al. (2017) Myocardial bridge as a cause of pseudo-Wellens' syndrome. Hellenic J Cardiol [Crossref]

7. Epstein AE, DiMarco JP, Ellenbogen KA, Estes NA, Freedman RA, et al. (2008) ACC/AHA/HRS 2008 Guidelines for Device-Based Therapy of Cardiac Rhythm Abnormalities: a report of the American College of Cardiology/American Heart Association Task Force on Practice Guidelines (Writing Committee to Revise the ACC/ AHA/NASPE 2002 Guideline Update for Implantation of Cardiac Pacemakers and Antiarrhythmia Devices): developed in collaboration with the American Association for Thoracic Surgery and Society of Thoracic Surgeons. Circulation 117: e350-408. [Crossref]

8. Lader JM, Park D, Aizer A, Holmes D, Chinitz, A, et al. (2018) Slow pathway modification for treatment of pseudo-pacemaker syndrome due to first-degree atrioventricular block with dual atrioventricular nodal physiology. HeartRhythm Case Rep 4: 98-101. [Crossref]

9. Epstein AE, DiMarco JP, Ellenbogen KA (2013) American College of Cardiology Foundation: AHA task force on practice guidelines: Heart rhythm society. Circulation 127: $3283-\mathrm{e} 352$

10. Pseudo Infarctions (2011) Circulation 03: e69.

11. Khan IA (2002) Wolff-Parkinson-White syndrome: pseudo myocardial infarction and ventricular hypertrophy. BMJ 324:662.

12. Tannemaat MR, Thijs RD, van Dijk JG (2014) Managing psychogenic pseudosyncope: Facts and experiences. Cardiol J 21: 658-664. [Crossref]

13. Kuga K, Li A, Endo M, Niho B, Suzuki A, et al. (2002) Atrial components contributing to pseudo $\mathrm{r}^{\prime}$ deflection in lead V1 in slow/fast atrioventricular nodal reentrant tachycardia: analysis of the atrial activation sequence by basket catheter isochronal mapping. Circ J 66: 236-240. [Crossref]

14. Schaeffer JW, Pryor R (1977) Pseudo left axis deviation and the S1S2S3 syndrome in chronic airway obstruction. Chest 71: 453-455. [Crossref]

15. Marcus GM, Atwood JE (2003) Images in clinical medicine. "T-U-P" syndrome, or pseudoatrial flutter. $N$ Engl J Med 349: e15. [Crossref]

16. Nam MC, Best L, Greaves K, Dayananda N (2016) Global pseudo-atrial flutter ECG appearance secondary to unilateral parkinsonian tremors. BMJ Case Rep pii: bcr2015214048. [Crossref]

Copyright: (C2018 Mishra A. This is an open-access article distributed under the terms of the Creative Commons Attribution License, which permits unrestricted use, distribution, and reproduction in any medium, provided the original author and source are credited. 